\title{
Equation of state for SU(3) gauge theory with RG improved action*
}

\author{
CP-PACS Collaboration: A. Ali Khan, ${ }^{\mathrm{a}}$ S. Aoki, ${ }^{\mathrm{b}}$ R. Burkhalter, ${ }^{\mathrm{a}, \mathrm{b}}$ S. Ejiri, ${ }^{\mathrm{a}}$ M. Fukugitac,

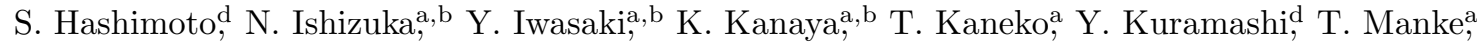 \\ K. Nagai, M. Okamoto, M. Okawa, A. Ukawa, ${ }^{\mathrm{a}, \mathrm{b}}$ and T. Yoshiée ${ }^{\mathrm{a}, \mathrm{b}}$ \\ ${ }^{a}$ Center for Computational Physics, University of Tsukuba, Tsukuba, Ibaraki 305-8577, Japan \\ bInstitute of Physics, University of Tsukuba, Tsukuba, Ibaraki 305-8571, Japan \\ ${ }^{\mathrm{c}}$ Institute for Cosmic Ray Research, University of Tokyo, Tanashi, Tokyo 188-8502, Japan \\ ${ }^{\mathrm{d}}$ High Energy Accelerator Research Organization (KEK), Tsukuba, Ibaraki 305-0801, Japan
}

\begin{abstract}
We present results for the equation of state for pure $\mathrm{SU}(3)$ gauge theory obtained with a renormalization-group (RG) improved action. The energy density and pressure are calculated on a $16^{3} \times 4$ and a $32^{3} \times 8$ lattice employing the integral method. Extrapolating the results to the continuum limit, we find the energy density and pressure to be in good agreement with those obtained with the standard plaquette action within the error of $3-4 \%$.
\end{abstract}

\section{Introduction}

The study of thermodynamic properties of QCD is crucial for understanding the early Universe and relativistic heavy-ion collisions [1]. The data is encapsulated in the equation of state (EOS). Recently the Bielefeld group determined the EOS in the continuum limit for pure gauge theory using the standard plaquette action 2]. Extending this result to full QCD will require the use of improved actions to compensate the increased computer power necessary for full QCD simulations. As a first step of such a program, we have studied the EOS for pure gauge theory with a renormalization-group (RG) improved action[3]. A summary of results [4] is presented in this article.

\section{Simulation parameters}

The RG-improved action we use has the form [3] $S_{g}=c_{0} \sum(1 \times 1$ loop $)+c_{1} \sum(1 \times 2$ loop $)$ with $c_{0}=1-8 c_{1}$ and $c_{1}=-0.331$.

We perform simulations on $16^{3} \times 4$ and $32^{3} \times 8$ lattices, and also on symmetric $16^{4}$ and $32^{4}$ lattices, at about 10 values of $\beta=6 / g^{2}$ in the range $T / T_{c} \approx 0.9-3.5$. We generate 20000 to 36000 iterations after thermalization on asym-

\footnotetext{
*Talk presented by M. Okamoto
}

metric lattices, and about 10000 iterations on symmetric lattices, where one iteration consists of one pseudo-heat-bath sweep followed by four over-relaxation sweeps. Errors are determined by the jack-knife method.

\section{Temperature scale and critical temper- ature}

We fix the temperature scale using the string tension of the static quark potential: $\frac{T}{T_{c}}=$ $\frac{(a \sqrt{\sigma})\left(\beta_{c}\right)}{(a \sqrt{\sigma})(\beta)}$. For this purpose, we fit results for $a \sqrt{\sigma}$ [5] using a scaling ansatz proposed by Allton[6],

$(a \sqrt{\sigma})(\beta)=f(\beta)\left(1+c_{2} \hat{a}(\beta)^{2}+c_{4} \hat{a}(\beta)^{4}\right) / c_{0}$

where $f(\beta)$ is the two-loop scaling function of $\mathrm{SU}(3)$ gauge theory, and power corrections in the pseudo lattice spacing $\hat{a}(\beta) \equiv \frac{f(\beta)}{f\left(\beta_{1}\right)}$ are introduced to incorporate deviations from two-loop scaling, with $\beta_{1}$ an arbitrary reference point.

In Fig. 1, we plot the critical temperature in units of the string tension, $T_{c} / \sqrt{\sigma}=$ $1 /\left(N_{t} a \sqrt{\sigma}\left(\beta_{c}\right)\right)$, for the RG-improved action together with the result for the standard plaquette action [7]. Making a quadratic extrapolation in $1 / N_{t}$, we find $T_{c} / \sqrt{\sigma}=0.650(5)$ in the continuum limit for the RG-improved action, which is $3 \%$ higher than the value $0.630(5)$ for the stan- 


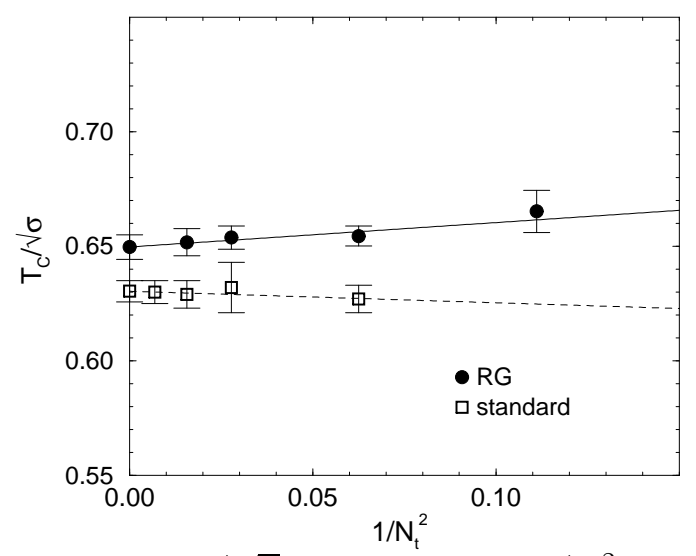

Figure 1. $T_{c} / \sqrt{\sigma}$ as a function of $1 / N_{t}^{2}$.

dard plaquette action[7]. The discrepancy may be caused by systematic uncertainties in the determination of the string tension for the two actions, which differ in the details.

\section{Equation of state}

\subsection{Integral method}

We calculate the energy density $\epsilon$ and pressure $p$ using the integral method [8]:

$$
\left.\frac{p}{T^{4}}\right|_{\beta_{0}} ^{\beta}=\int_{\beta_{0}}^{\beta} \mathrm{d} \beta^{\prime} \Delta S, \quad \frac{\epsilon-3 p}{T^{4}}=T \frac{\mathrm{d} \beta}{\mathrm{d} T} \Delta S .
$$

Here $\Delta S \equiv N_{t}^{4}\left(\langle S\rangle_{T}-\langle S\rangle_{0}\right)$, where $\langle S\rangle_{T}$ and $\langle S\rangle_{0}$ are the expectation values of the action density $S=S_{g} / N_{s}^{3} N_{t}$ at finite and zero temperature, respectively. The beta function $d \beta / d T$ is determined from the scale parametrized by (11).

Our results for the pressure $p$ are shown in Fig.2 together with those from the standard action. We find that the magnitude of the cut-off dependence for the RG-improved action is similar to that for the standard action, and opposite in sign.

We extrapolate the energy density and pressure to the continuum limit, assuming a quadratic dependence on $1 / N_{t}$ as the RG-improved action has discretization errors of $O\left(a^{2}\right)$. In Fig. 3, results of the extrapolation are plotted by solid lines, and are compared with those for the standard plaquette action (dot-dashed lines). As observed in this figure, results in the continuuum limit for the two actions are in good agreement with each other

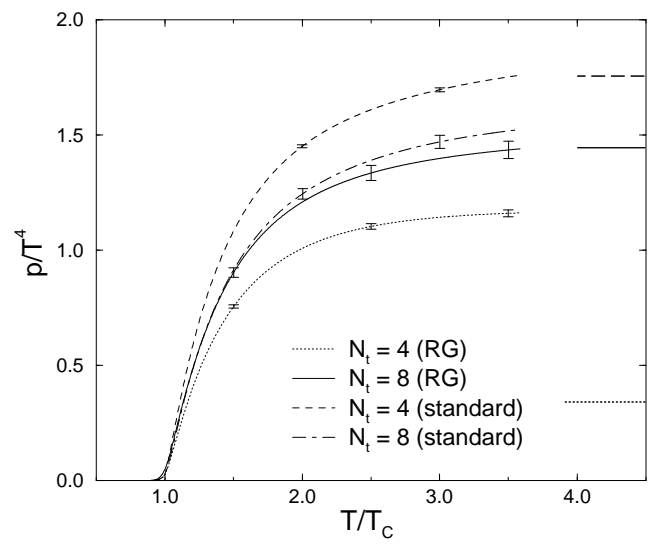

Figure 2. Pressure for $N_{t}=4$ and 8.

within the estimated error of 3-4\%.

We note that the curves for the plaquette action in Fig. 3 are obtained from a reanalysis of the data in Ref. [2] employing the same ansatz for the string tension (11) in order to avoid ambiguities arising from the choice of scale. In practice we found the changes in the pressure and energy density due to the choice of scale to be very small, being less than the statistical error of $1-3 \%$.

\subsection{Operator method}

The energy density can also be calculated through the operator method [9]:

$\frac{\epsilon}{T^{4}}=\frac{18 N_{t}^{4}}{g^{2}}\left[c_{s}\left(\left\langle S_{s}\right\rangle-\langle S\rangle_{0}\right)-c_{t}\left(\left\langle S_{t}\right\rangle-\langle S\rangle_{0}\right)\right](3)$

where $c_{s}$ and $c_{t}$ are the asymmetry coefficients. The pressure can then be obtained with the second equation of (2).

In Fig. 4 we compare results obtained with the integral and operator methods. The one-loop perturbative values 10] are used for the asymmetry coefficients. We observe that the two sets of results are consistent with each other. The remaining discrepancy may well arise from the use of one-loop asymmetry coefficients; for the plaquette action, non-perturbative effects are known to be important 11.

In the high-temperature limit one may calculate the EOS by perturbation theory. The leading-order results for the EOS are shown by horizontal lines in Fig. A. As has been noted some time ago[12], the perturbative value for $N_{t}=4$ is 


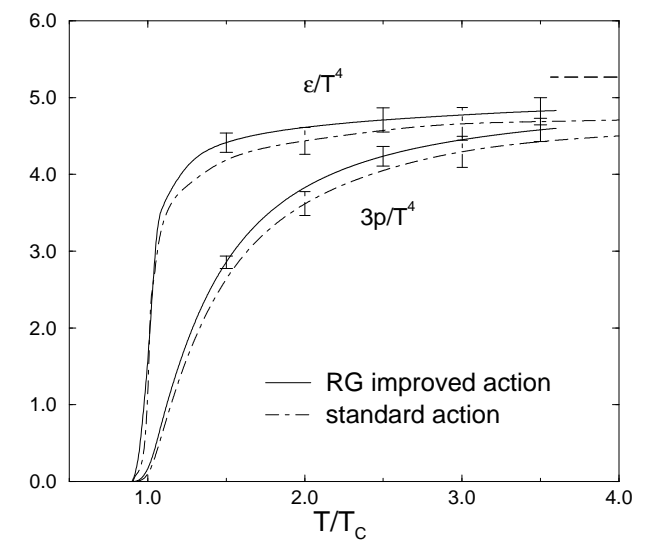

Figure 3. Equation of state in the continuum limit for the RG-improved action (solid lines) and for the standard action (dash-dotted lines).

very small for the RG-improved action. Our numerical results are much larger than this value, at least in the range $T / T_{c} \lesssim 3.5$ explored in the present work.

A possible source of the discrepancy is a breakdown of perturbation theory due to the infrared divergence. Another possibility is that pressure and energy density decrease towards the perturbative values at high temperatures. This, however, would require an unusual situation of a negative $\Delta S$ since the pressure is expressed as an integral of $\Delta S$ with the integral method.

\section{Conclusions}

Our continuum result for the EOS of pure $\mathrm{SU}(3)$ gauge theory obtained with an RGimproved gauge action shows a good agreement with that from the plaquette action. This provides a concrete support for the expectation that continuum results are insensitive to the choice of lattice actions. We also found that the energy density and pressure for $N_{t}=4$ overshoot the perturbative high temperature limit. Understanding the origin of this behavior is left for future investigations.

This work is supported in part by the Grantsin-Aid of Ministry of Education, Science and Culture (Nos. 09304029, 10640246, 10640248,

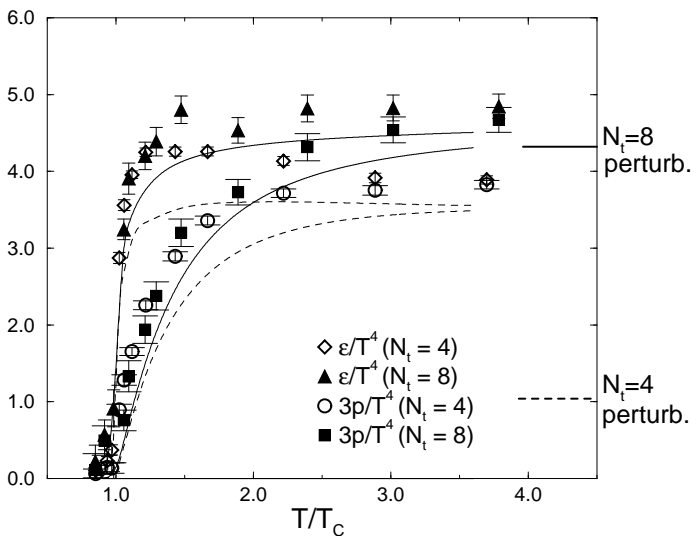

Figure 4. EOS from the operator method using one-loop values for the asymmetry coefficients as compared with those from the integral method. Dashed and solid horizontal lines are perturbative high temperature limit at $N_{t}=4$ and 8 , which is common for $\epsilon / T^{4}$ and $3 p / T^{4}$.

10740107, 11640250, 11640294, 11740162). SE, KN and M. Okamoto are JSPS Research Fellows. AAK and TM are supported by the Research for the Future Program of JSPS.

\section{REFERENCES}

1. For a review, F. Karsch, these proceedings.

2. G. Boyd et al., Nucl. Phys. B469 (1996) 419.

3. Y. Iwasaki, Nucl. Phys. B258 (1985) 141; Univ. of Tsukuba report UTHEP-118 (1983).

4. CP-PACS Collaboration, M. Okamoto et al., hep-lat/9905005, to appear in Phys. Rev. D.

5. Y. Iwasaki et al., Phys. Rev. D56 (1997) 151; CP-PACS Collaboration, in preparation.

6. C. Allton, hep-lat/9610016.

7. B. Beinlich et al., Eur. Phys. J. C6 (1999) 133.

8. J. Engels et al., Phys. Lett. B252 (1990) 625.

9. J. Engels et al., Phys. Lett. B101 (1981) 89.

10. S. Sakai et al., Nucl. Phys. B (Proc. Suppl.) 73 (1999) 417; S. Sakai, private communucation.

11. S. Ejiri et al., Phys. Rev. D58 (1998) 094505; J. Engels et al., hep-lat/9905002.

12. F. Karsch, Nucl. Phys. B (Proc. Suppl.) 60A (1998) 169. 